\title{
Baicalein regulates NEDD4L-mediated TLR2 ubiquitination to relieve Mycobacterium tuberculosis-induced pneumonia in mice
}

\author{
Min SHI ${ }^{1}$, Pengyi YIN ${ }^{2}$, Xiaobo GUO ${ }^{3}$, Qian $\mathrm{LI}^{1}$, Lin SUN ${ }^{1}$, Xiaohua CAO ${ }^{4 *}$
}

\begin{abstract}
Baicalein has strong anti-inflammatory activity, but its efficacy and mechanism in pneumonia of mice is still unclear. Herein, RAW264.7 cells were infected with $M$. tuberculosis H37Rv (MOI 1:10) after pre-incubation with $50 \mu \mathrm{M}$ baicalein, and the result found that baicalein incubation ameliorated the increase of bacterial load, and reduced the levels of ROS, IL-12p40, TLR2 protein, phosphorylated JNK protein and M1 polarization markers (iNOS and CD11c), and increased the levels of IL-10, M2 polarization markers (Arg1, Mrc1 and CD206) and NEDD4L protein in H37Rv infected-RAW264.7 cells. And lentivirus-mediated NEDD4L overexpression vector (LV-NEDD4L) transfection had the same effect as baicalein, while small interfering RNA target NEDD4L (si-NEDD4L) transfection or JNK pathway inhibitor IQ 3 treatment reversed the effects of baicalein, and ubiquitin inhibitor MG132 treatment reversed the effects of LV-NEDD4L. Co-immunoprecipitation (Co-IP) and TLR2 ubiquitination assays found that the binding of NEDD4L and TLR2 promoted the ubiquitination of TLR2. In vivo, C57BL/6 mice were nasal infected with $\mathrm{H} 37 \mathrm{Rv}(1000 \pm 150 \mathrm{CFU} /$ mouse) to establish a mouse pneumonia model, and then administered with baicalein. The result found that baicalein improved H37Rv-induced pneumonia in mice. In conclusion, baicalein increased NEDD4Lmediated ubiquitination of TLR2, promoting macrophage M2 polarization and alleviating $\mathrm{H} 37 \mathrm{Rv}$-induced pneumonia.
\end{abstract}

Keywords: Mycobacterium tuberculosis; baicalein; NEDD4L; TLR2; pneumonia.

Practical Application: Baicalein has strong anti-inflammatory activity, but its efficacy and mechanism in pneumonia of mice caused by $M$. tuberculosis is still unclear. Here, we find that baicalein treatment alleviates mouse pneumonia caused by $M$. tuberculosis $\mathrm{H} 37 \mathrm{Rv}$ in vivo and H37Rv-infected RAW264.7 cells dysfunction in vitro. Mechanism investigation reveals that baicalein could increase NEDD4L-mediated ubiquitination of TLR2 by increasing the expression level of NEDD4L protein, alleviating the inflammatory environment, promoting macrophage M2 polarization, thereby alleviating $\mathrm{H} 37 \mathrm{Rv}$-induced pneumonia in mice.

\section{Introduction}

Mycobacterium tuberculosis (M. tb) does not develop immediately after infection, but manifests itself in a clinically asymptomatic state called the incubation period of tuberculosis (TB) infection. About 22 to $32 \%$ of the world's population is in this state. Although less than $10 \%$ of the population eventually suffers from the disease, tuberculosis is still the chief culprit in deaths among infectious diseases (Houben \& Dodd, 2016). As we all know, TB mainly infects the lungs, and primary TB is mainly spreads through the bronchi to cause caseous pneumonia, and then caseous pneumonia either ruptures to create a cavity, or stays in and causes primary granuloma and fibrous case-like disease, leading to pulmonary fibrosis and necrosis (Hunter, 2018). It is reported that Chinese medicine can significantly alleviate the decline in lung function and the production of alveolar inflammatory factors caused by various stimuli (Huang et al., 2021). Baicalein is one of the flavonoids with the highest content in the traditional Chinese medicine Scutellaria, and it has strong anti-inflammatory activity (Dinda et al., 2017). It is reported that baicalin can relieve acute respiratory distress syndrome in rats by reducing the secretion of alveolar inflammatory factors and improving lung function (Bao et al., 2021). A report claimed that baicalein can protect mice from death caused by Streptococcus pneumoniae by reducing alveolar capillary membrane permeability, lung infiltration of inflammatory cells, inflammatory cytokine production and lung cell apoptosis. But the effect of baicalein on $M$. $t b$ has not been reported yet.

$M . t b$ mainly exists in antigen-presenting cells in the host, including macrophages and dendritic cells, and evades the body's immune defenses by inhibiting the secretion of MHC class II molecules and preventing antigen presentation (Repique et al., 2003). In this process, the glycolipids on the cell wall surface of M. tb, including lipoarabinomannan (LAM), lipomannan (LM) 
and phosphatidylinositol mannoside (PIM), bind to toll-like receptor 2 (TLR2) to inhibit interleukin-10 (IL-10) secretion to prevent the maturation of macrophages (Gilleron et al., 2006; Harding \& Boom, 2010). It is reported that baicalein can detoxify hepatic sinusoidal obstruction syndrome by reducing the content of malondialdehyde (MDA), the expression of TLR2 and the activation of nuclear factor $\mathrm{\kappa B}(\mathrm{NF}-\mathrm{\kappa B})$ transcription in the liver of rats induced by monocrotaline (Zhang et al., 2016). In addition, baicalein can reduce the secretion of inflammatory factors and cell apoptosis by reducing the expression of TLR2 protein, and alleviate nerve cell damage caused by oxygen and glucose deprivation (Li et al., 2012). However, the mechanism by which baicalein regulates TLR2 protein expression is still unclear.

In cells, protein degradation is achieved through different degradation systems, among which the ubiquitin proteasome system (UPS) participates in the degradation of most cell proteins, and regulates various cell functions by degrading more than $80 \%$ of cell proteins to regulate the key to various cell functions, including cell cycle, cell survival and apoptosis, gene transcription and translation and cell metabolism (MeyerSchwesinger, 2019). Protein ubiquitination is a continuous ATP consumption process involving E1, E2 and E3 ubiquitin ligases, it mediates the covalent attachment of one or more ubiquitin proteins to the target protein and this coupling mode leads to the target protein degradation, affecting its activity or changing its location (Collins \& Goldberg, 2017). According to a report, E3 ubiquitin ligase TRIM58 can ubiquitination degrade TLR2 by binding to TLR2, negatively regulate TLR 2 signaling in human bone marrow-like cell lines, and inhibit intestinal mucosal inflammation (Eyking et al., 2019). It is reported that baicalein can induce histone deacetylase degradation through the ubiquitin proteasome pathway, inhibit the growth of acute myeloid leukemia cell lines and induce their differentiation (Yu et al., 2020).

Neural precursor cell expressed developmentally downregulated gene 4-like (NEDD4L) is an E3 ubiquitin ligase. A study showed that in silver nanoparticles-induced mouse pneumonia, the expression level of NEDD4L mRNA is significantly downregulated, and it is significantly negatively correlated with the influx of neutrophils (Scoville et al., 2017), and NEDD4L may be a candidate gene to regulate pneumonia in mice. Moreover, in a genomic study on cardiovascular disease, it was found that both NEDD4L and TLR2 are associated with the ischemic heart disease phenotype (Makeeva et al., 2015). Therefore, we speculate that there may be a regulatory relationship between NEDD4L and TLR2. In this study, we investigate the effects of baicalein on pneumonia in C57BL/6 mice and RAW264.7 cells polarization caused by $M . t b \mathrm{H} 37 \mathrm{Rv}$ infection in vivo and in vitro, and the corresponding mechanisms.

\section{Materials and methods}

\subsection{Animal studies}

Male C57BL/6 mice $(\mathrm{n}=30)$ about 8 weeks old, weighing 22-24 g, were purchased from the Experimental Animal Center of Xian Jiaotong University Medical College. The 4 mice were housed together in a cage $(27 \times 17 \times 13 \mathrm{~cm})$ under a light-dark cycle (light on at 20:00 and light off at 08:00) environment. All mice eat and drink freely. The experimental procedures were followed the guidelines of KUMW for the care and use of animals, and were approved by the Animal Care and Use Committee of Xi'an Peihua University Medical School Medical Welfare Institution. All mice were randomly divided into 3 groups: control group $(\mathrm{n}=10), \mathrm{H} 37 \mathrm{Rv}$ infection group $(\mathrm{n}=10)$ and baicalein treatment group $(\mathrm{n}=10)$. After 7 days of adaptive feeding, mice in the $\mathrm{H} 37 \mathrm{Rv}$ infection group were intranasal infected with $M . t b \mathrm{H} 37 \mathrm{Rv}$ in 0 th and 7th days with $1000 \pm 150$ $\mathrm{CFU} /$ mouse after intraperitoneal infected light ketamine (100 $\mathrm{mg} / \mathrm{kg})$-xylasine $(10 \mathrm{mg} / \mathrm{kg})$ anesthesia, after that, the mice were intraperitoneally injected with an equal volume of $0.9 \%$ normal saline every other day until the 28 th day. The mice in the control group were only anesthetized and injected intraperitoneally with an equal volume of $0.9 \%$ saline. And mice in the baicalein treatment group were injected intraperitoneally with $25 \mathrm{mg} / \mathrm{kg}$ baicalein (B107323, aladdin, Beijing, China) every other day till the 28th day after infection with $M$. $t b \mathrm{H} 37 \mathrm{Rv}$. For quantifying bacterial burden at days 28 post-infection, lungs were weighted, defined aliquots homogenized in PBS, and tenfold serial dilutions prepared in $0.05 \%$ Tween 20 containing $0.9 \% \mathrm{NaCl}$ were plated in duplicates onto Middlebrook 7H11 (Gibco) agar plates containing $10 \%$ OADC. After 3 weeks incubation at $37^{\circ} \mathrm{C}$ colonies were enumerated, and results expressed as $\log _{10} \mathrm{CFU}$ per organ. The animal experiment design is shown in Figure 1.

\subsection{Cell lines and culture}

Mouse mononuclear macrophage leukemia cell line RAW264.7 (EY-X0708, ATCC, USA) and human embryonic kidney cell line HEK-293T (CL-0005, Procell, China) were cultured at $37^{\circ} \mathrm{C}$ in $5 \% \mathrm{CO}_{2}$ incubator (Thermo Fisher Scientific, Waltham, MA, USA) in DMEM medium (Gibco, Rockville, MD), supplemented with $10 \%$ FBS (IBM, Almon, NY), $100 \mathrm{U} / \mathrm{mL}$ penicillin and $100 \mu \mathrm{g} / \mathrm{mL}$ streptomycin. RAW264.7 cells were transfected with lentivirusmediated NEDD4L overexpression vector (LV-NEDD4L), small interfering RNA target NEDD4L (si-NEDD4L) and corresponding negative control named as Vector or Scramble for $24 \mathrm{~h}$, which were constructed via technical support from Sangon Biotech

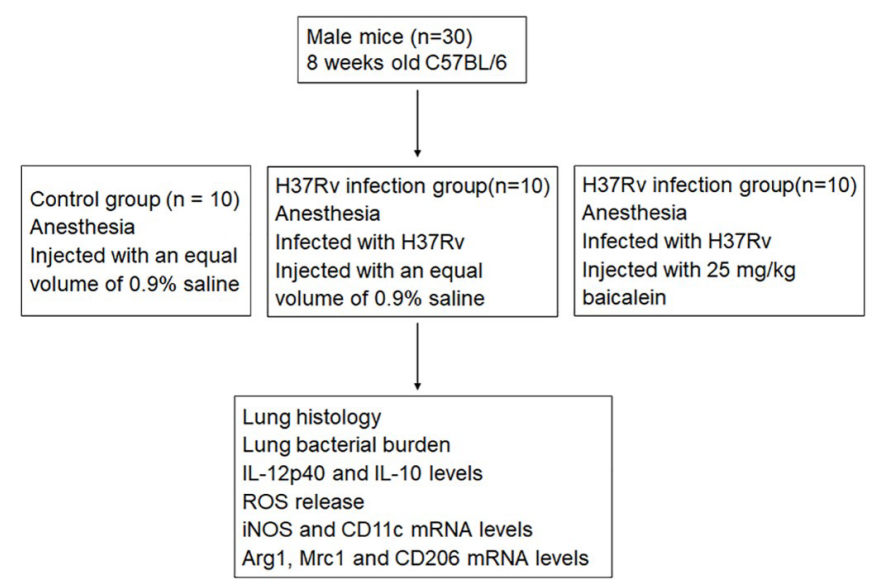

Figure 1. Experimental design of baicalein alleviating lung injury in mice induced by Mycobacterium tuberculosis H37Rv infection. 
(Shanghai, China), following the NYMC Institutional Biosafety Committee approval. $50 \mu \mathrm{M}$ baicalein (aladdin) was incubated with cells for $24 \mathrm{~h}$ before injection with $M$. $t b \mathrm{H} 37 \mathrm{Rv}$, and then $1.4 \mu \mathrm{M}$ JNK pathway inhibitor IQ 3 was added and incubated with cells for $6 \mathrm{~h}$, proceeded to the next experiment.

\subsection{Mycobacterium tuberculosis culture and infection}

M. tb virulent strain $\mathrm{H} 37 \mathrm{Rv}$ (ATCC) was stored in a refrigerator at $-80^{\circ} \mathrm{C}$. After thawing the bacterial solution, it was added to Middlebrook 7H9 broth medium (Shanghai Canspec, China) and cultured in a horizontal shaker at $37^{\circ} \mathrm{C}$. After a series of treatments, RAW264.7 cells were infected with $M$. tb H37Rv (MOI 1:10) for 4-6 h, washed with PBS 5-6 times to remove the $M . t b \mathrm{H} 37 \mathrm{Rv}$ outside the cells, and then added fresh DMEM solution to continue culturing for a specified time before testing.

\subsection{Plasmid construction and cell transfection}

Flag-TLR2, HA-Ubiquitin (WT), and Myc-NEDD4L were constructed by Sangon Biotech. And 293T cells transfection was performed using Lipofectamine 2000 (Invitrogen) according to the manufacturer s instructions. The pcDNA3.1 was obtained from Thermo Fisher Scientific (Waltham, MA, USA). TLR2 and NEDD4L were cloned into XhoI site of pcDNA-Flag and pcDNA-MYC vectors. pRK5-Flag-TLR2, pRK5-MYC-NEDD4L, pRK5-HA-ubiquitin were constructed via technical support from Sangon Biotech (Shanghai, China).

\subsection{Reverse Transcriptionquantitative PCR (RTqPCR)}

Total RNA from tissues and cells was extracted with Trizol reagent (Invitrogen, Carlsbad, CA, USA) under the manufacturer's instructions. Then, the extracted RNAs were reversely transcribed to be cDNA with PrimeScript RT reagent Kit (BioTeke, Beijing, China). RT-qPCR was performed with SYBR Premix Ex Taq Kit (BioTeke, Beijing, China) on an ABI 7500 system (Applied Biosystems, Foster City, CA, USA). The PCR parameters were performed at $95^{\circ} \mathrm{C}$ for $1 \mathrm{~min}$, followed by 30 circles at $55^{\circ} \mathrm{C}$ for $30 \mathrm{~s}$ and $72{ }^{\circ} \mathrm{C}$ for $90 \mathrm{~s}$. GAPDH was served as an internal reference. Data were analyzed by using the $2^{-\Delta \Delta C T}$ method. The following primers were used: inducible nitric oxide synthase (iNOS, forward $5^{\prime}-3^{\prime}$ : GGC AGC CTG TGA GAC CTT TG; reverse $5^{\prime}-3^{\prime}$ : GAA GCG TTT CGG GAT CTG AA); CD11c (forward $5^{\prime}-3^{\prime}$ : CTG GAT AGC CTT TCT TCT GCT G; reverse $5^{\prime}-3^{\prime}$ : GCA CAC TGT GTC CGA ACT C); CD206 (forward 5'-3': TCA GCT ATT GGA CGC GAG GCA; reverse 5'-3': TCC GGG TTG CAA GTT GCC GT); mouse arginase-1 (Arg1, forward 5'-3': CAG AAG AAT GGA AGA GTC AG; reverse $5^{\prime}-3^{\prime}$ : CAG ATA TGC AGG GAG TCA $\mathrm{CC}$ ); mouse mannose receptor $\mathrm{C}$ type 1 (Mrc1, forward $5^{\prime}-3^{\prime}$ : TTC GGG ATT GTG GAG CAG ATC; reverse5'-3': TTG TCG TAG TCA GTG GTG GTT C) and mouse glyceral-dehyde-3phosphate dehydrogenase (GAPDH, forward 5' $-3^{\prime}$ : GAG CCA AAC GGG TCA TCA TCT; reverse 5' -3': GAG GGG CCA TCC ACA GTC TT).

\subsection{Western blot analysis}

Proteins extracted from cells and tissues were treated with RIPA lysis and extraction buffer (Sigma, St. Louis, MO, USA). The BCA protein assay kit (Sigma) was used to quantify the protein, and $25 \mu \mathrm{g}$ protein from each sample was separated by SDS-PAGE and transferred to PVDF membranes (Millipore, Billerica, MA, USA). After being blocked with $5 \%$ nonfat milk solution for $1 \mathrm{~h}$, the membranes were incubated with the following primary antibodies (Abcam, Cambridge, UK) at $4{ }^{\circ} \mathrm{C}$ overnight: Flag (1:500; ab205606), Myc (1:1000; ab32072), HA (1:5000; ab9110), NEDD4L (1:1000; ab46521), TLR2 (1:1000; ab209217), JNK (1:2500; ab199380), p-JNK (1:2000; ab124956) and $\beta$-actin (ab8227; 1:1000). Then the membrane was probed with a secondary antibody labeled with goat anti-rabbit horseradish peroxide at room temperature for $1 \mathrm{~h}$. The protein bands were visualized and analyzed by using ECL-Plus Western Blot analysis and detection system (Thermo Fisher Scientific, Waltham, Massachusetts, USA). $\beta$-actin was set as a loading control.

\subsection{Enzyme-Linked Immunosorbent Assay (ELISA)}

Cell culture supernatant and lung-homogenate were collected, and then ELISA kits (Qiagen, Dusseldorf, Germany) were used to determine the concentrations of IL-12p40 and IL-10 according to the standard instructions. The experimental data were recorded and the contents of inflammatory factors in cells and tissues were calculated.

\subsection{Detection of ROS content by flow cytometry}

Cells were incubated with $10 \mathrm{mM}$ DCF-DA for $30 \mathrm{~min}$ in the dark at $37^{\circ} \mathrm{C}$. Then cells were washed with PBS for 3 times. And the cells were digested with trypsin (without ethylenediaminetetraacetic acid). The level of intracellular ROS was detected in the FITC-A channel of a FACScan flow cytometer (Becton Dickinson, NJ, USA), using $488 \mathrm{~nm}$ excitation and 525 $\mathrm{nm}$ emission. The number of stained cells was calculated using Cell Quest software (Becton Dickinson).

\subsection{Proteasome inhibition test}

RAW264.7 cells were cultured in DMEM medium containing $10 \%$ fetal bovine serum; cells were digested with $0.25 \%$ trypsin for $5 \mathrm{~min}$ and passaged at 1:5. And then 10,20, 50 and $100 \mu \mathrm{M}$ baicalein were added to the culture medium and incubated with the cells for $24 \mathrm{~h}$ in $37^{\circ} \mathrm{C}$ incubator containing $5 \% \mathrm{CO}_{2}$, and $20 \mu \mathrm{M}$ proteasome inhibitor MG-132 was added, and cells were collected $4 \mathrm{~h}$ later.

\subsection{Co-Immunoprecipitation experiment (Co-IP)}

Co-IP experiments were carried out in HEK293T cells. In brief, cells were transfected with indicated constructs for $24 \mathrm{~h}$, and then lysed in a cell lysis buffer $(20 \mathrm{mM}$ Tris- $\mathrm{HCl}, \mathrm{pH} 7.5,150$ $\mathrm{mM} \mathrm{NaCl}, 1 \%$ Triton X-100, $0.5 \mathrm{mM}$ EDTA, protease inhibitor cocktail (Roche, Basel, Switzerland) and phosphatase inhibitor cocktail (Roche)). After centrifugation, the supernatants were pulled-down by indicated antibodies and then analyzed by immunoblotting. For analysis of endogenous protein complexes in post-osteogenic $\mathrm{C} 3 \mathrm{H} 10 \mathrm{~T} 1 / 2$ cells, nuclear extracts from $5 \times 10^{7}$ 
cells were collected in $1 \mathrm{~mL}$ of buffer containing $20 \mathrm{mM}$ Tris$\mathrm{HCl}, \mathrm{PH}$ 7.4, $300 \mathrm{mM} \mathrm{NaCl}, 1 \%$ Triton X-100, 2 mM EDTA, protease inhibitor cocktail, and phosphatase inhibitor cocktail.

\subsection{Ubiquitination analysis}

Indicated constructs were co-transfected into HEK293T cells along with HA-ubiquitin for $24 \mathrm{~h}$, and then cells were harvested in a denaturing buffer $(6 \mathrm{M}$ guanidine- $\mathrm{HCl}, 0.1 \mathrm{M}$ $\mathrm{Na} 2 \mathrm{HPO} 4 / \mathrm{NaH} 2 \mathrm{PO} 4, \mathrm{pH} 8.0,10 \mathrm{mM}$ imidazole). The lysates were incubated with Ni-nitrilotriacetic acid (Ni-NTA) agarose beads for 3 hours, followed by four times washes with the denaturing buffer and two times with a low-salt buffer $(25 \mathrm{mM}$ Tris- $\mathrm{HCl}, \mathrm{pH}$ 6.8, $20 \mathrm{mM}$ imidazole). Ubiquitinated proteins were eluted by boiled in the SDS-sample buffer in the presence of $200 \mathrm{mM}$ imidazole. After centrifugation, the supernatants were analyzed by immunoblotting.

\subsection{Histopathological analysis}

Lung tissues were fixed in $4 \%$ buffered formaldehyde overnight, paraffin-embedded, and $3 \mathrm{~mm}$ sections were stained with hematoxylin and eosin (HE). Histologic changes were determined by using a semiquantitative severity score (0-5) for inflammatory cell infiltration and granuloma (0-5). The slides were examined blindly with a Nikon microscope (Nikon eclipse 80i; Nikon, Tokyo, Japan).

\subsection{Colony-Forming Unit (CFU) assay}

To assay bacterial viability, cells were infected with $M$. $t b$ $\mathrm{H} 37 \mathrm{Rv}$ at an MOI of 10 for $6 \mathrm{~h}$ at $37^{\circ} \mathrm{C}$, then washed 6 times with DMEM solution to remove any extracellular bacteria. Cells were collected at 6 or $24 \mathrm{~h}$ post challenge and the supernatant was rejected. The infected cells were lysed with sterile distilled water containing $0.06 \%$ SDS. Homogenates underwent 10 -foldserial dilution and each dilution was inoculated on $7 \mathrm{H} 11$ agar plates supplemented with $10 \%$ OADC and incubated at $37^{\circ} \mathrm{C}$ for 3-4 weeks. CFUs were calculated in triplicate using standard procedures, which was represented as $\log _{10} \mathrm{CFU} \pm$ SEM for each group $(n=6)$.

\subsection{Statistical analysis}

All experiments were repeated at least 3 times. Data from more than two independent experiments were reported as the mean \pm standard error of mean (SEM) and analyzed by using SPSS version 22.0. Statistical significance between two groups was evaluated with Student's t-test, and variation analysis was used for more than two-group comparison. A value of $P<0.05$ was considered statistically significant.

\section{Results}

\subsection{Baicalein promotes M2 type polarization of macrophages after Mycobacterium tuberculosis infection}

Macrophages are the main targets of $M$. tuberculosis infection. After M. tuberculosis infection, the first cause is the M1 polarization of macrophages, and the secretion of pro-inflammatory cytokines promote body damage. Then, the macrophages will undergo M2 polarization to repair the damage (Shi et al., 2019), and we asked whether baicalein affects the immune balance between M1 and M2 polarization of macrophages. We found that compared with the control group, the pro-inflammatory cytokine IL-12p40 level, the anti-inflammatory cytokine IL-10 level, ROS level and the mRNA levels of M1 polarization markers iNOS and CD11c were significantly increased (Figure 2A-D), and the mRNA levels of M2 polarization markers Arg1, Mrc1 and CD206 were significantly decreased (Figure 2E) in RAW264.7 cells in the $M$. $t b \mathrm{H} 37 \mathrm{Rv}$ infection group. In the baicalein treatment group, the IL-12p40 level, ROS level, the level of M1 polarization marker molecules and the colony count (Figure 2F) were significantly reduced, while the IL-10 level and the level of M2 polarization marker molecules were significantly increased in RAW264.7 cells compared with the $M . t b \mathrm{H} 37 \mathrm{Rv}$ infection group. And after baicalein treatment, the bacterial count of RAW264.7 cells at 6 and $24 \mathrm{~h}$ was significantly reduced (Figure $2 \mathrm{G}$ ), suggesting that baicalein may promote the M2 polarization of RAW264.7 cells after $M$. $t b$ infection.

\subsection{Upregulation of NEDD4L promotes M2 polarization of macrophages after Mycobacterium tuberculosis infection}

We found that the expression level of NEDD4L protein in RAW264.7 cells infected with $M$. tb H37Rv was significantly reduced (Figure $3 \mathrm{~A}$ ). To verify the effect of NEDD4L protein on the polarization of $\mathrm{H} 37 \mathrm{Rv}$-infected RAW264.7 cells, we overexpressed NEDD4L, and it was found that the expression level of NEDD4L protein was significantly increased, furthermore, IL-12p40 level (Figure 3B), ROS level (Figure 3D), M1 polarization marker molecules iNOS and CD11c mRNA levels were significantly reduced (Figure 3E), and IL-10 level (Figure 3C) and M2 polarization marker molecules Arg1, Mrc1 and CD206 mRNA levels were significantly increased (Figure $3 \mathrm{~F}$ ), and the bacterial count was significantly reduced (Figure $3 \mathrm{G}$ ). These results suggested that the upregulation of NEDD4L may promote the M2 polarization of RAW264.7 cells after H37Rv infection.

\subsection{Baicalein regulates the expression of NEDD4L to promote M2 type polarization of macrophages after Mycobacterium tuberculosis infection}

In the above studies, we found that both baicalein treatment and overexpression of E3 ubiquitin ligase NEDD4L could promote the M2 type polarization of RAW264.7 cells after $\mathrm{H} 37 \mathrm{Rv}$ infection. A previous study has shown that baicalein can regulate disease progression by activating the ubiquitin proteasome system (Yu et al., 2020). We speculate that baicalein may be related to the expression of NEDD4L protein. To our surprised, after baicalein treatment, the expression of NEDD4L protein in H37Rv-infected RAW264.7 cells was significantly increased (Figure 4A, B), while the expression of NEDD4L protein was significantly decreased after NEDD4L interference. Meanwhile, after NEDD4L interference, the levels of IL-12p40 and ROS were significantly increased (Figure 4C, E), and the level of IL-10 were significantly reduced (Figure 4D), and RAW264.7 cells M1 type polarization was significantly enhanced (Figure $4 \mathrm{~F}$ ), while the polarization of M2 was significantly weakened (Figure 4G). 
A<smiles>O=c1cc(-c2ccccc2)oc2cc(O)c(O)c(O)c12</smiles>

The chemical structure of baicalein

D

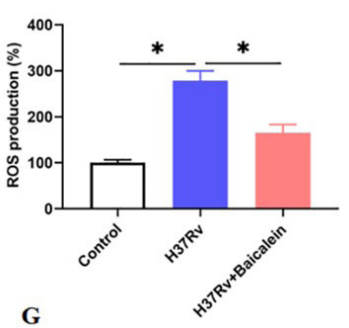

${ }_{10}^{10}$ ㅁ H37Rv $=$ H37Rv+Baicalein

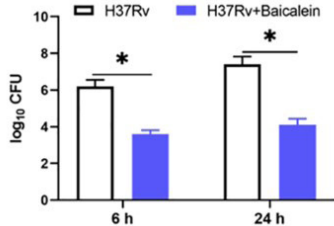

B

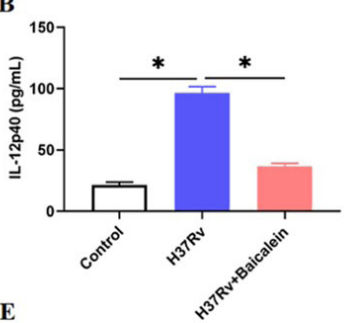

$\mathbf{E}$

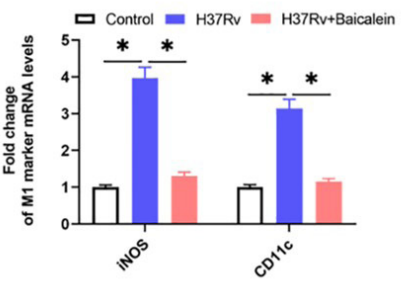

C
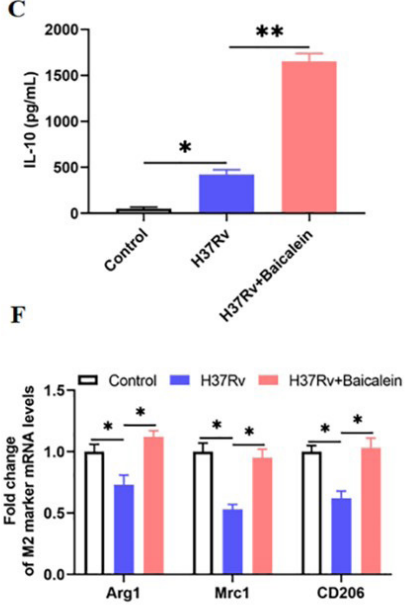

Figure 2. Baicalein promotes M2 type polarization of macrophages after Mycobacterium tuberculosis infection. RAW264.7 cells were infected with M. $t b \mathrm{H} 37 \mathrm{Rv}$ (MOI 1:10) for 4-6 h after pre-incubation with $50 \mu \mathrm{M}$ baicalein for $24 \mathrm{~h}$. (A) The chemical structure of baicalein; (B), (C) ELISA measurement of IL-12p40 and IL-10 in cells; (D) The ROS level was detected by flow cytometry (DCFH-DA); (E), (F) RT-qPCR analyzed the mRNA levels of M1 markers (iNOS and CD11c) and M2 markers (Arg1, Mrc1 and CD206); (G) Colony forming units (CFU) analyzed the infection of H37Rv. Statistical significance was analyzed utilizing the Student’s t-test. Values are exhibited as mean $\pm S E M$. N $=6,{ }^{\star} P<0.05,{ }^{* *} P<0.01$.

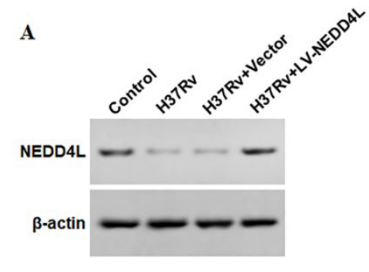

D

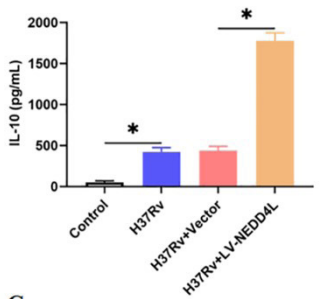

G

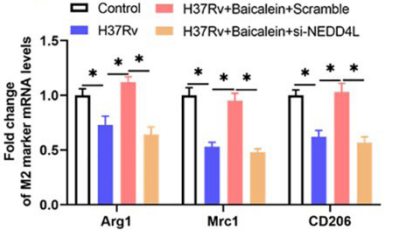

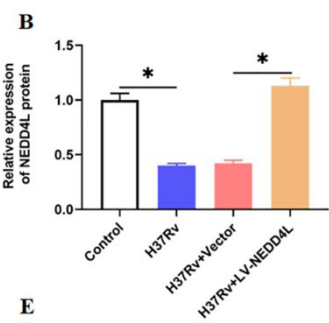

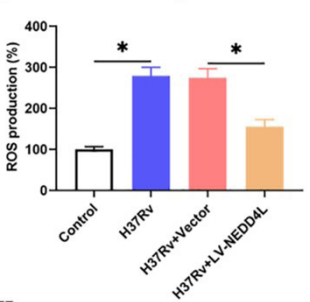

H

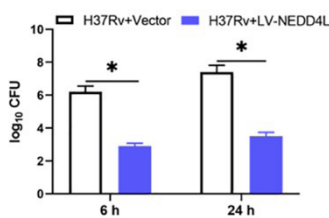

C

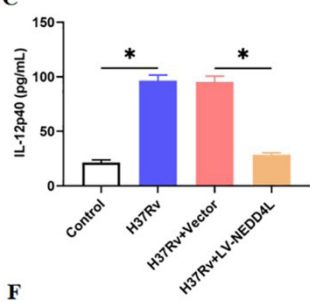

F Control $=$ H37Rv+vector

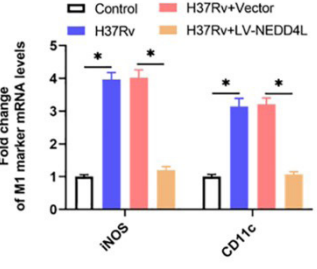

Figure 3. Up-regulation of NEDD4L promotes M2 polarization of macrophages after Mycobacterium tuberculosis infection. RAW264.7 cells were infected with $M . t b \mathrm{H} 37 \mathrm{Rv}$ (MOI 1:10) for 4-6 h after $24 \mathrm{~h}$ pre-transfection with the NEDD4L lentiviral overexpression vector (LV-NEDD4L) or the corresponding negative control vector (Vector). (A), (B) Western blotting was used to detect the protein expression of NEDD4L; (C), (D) ELISA measurement of IL-12p40 and IL-10 in cells; (E) The ROS level was detected by flow cytometry (DCFH-DA); (F), (G) RT-qPCR analyzed the mRNA levels of M1 markers (iNOS and CD11c) and M2 markers (Arg1, Mrc1 and CD206); (H) Colony forming units (CFU) analyzed the infection of H37Rv after transfection with LV-NEDD4L. Statistical significance was analyzed utilizing the Student's t-test. Values are exhibited as mean \pm SEM. $\mathrm{N}=6,{ }^{\star} P<0.05$. 

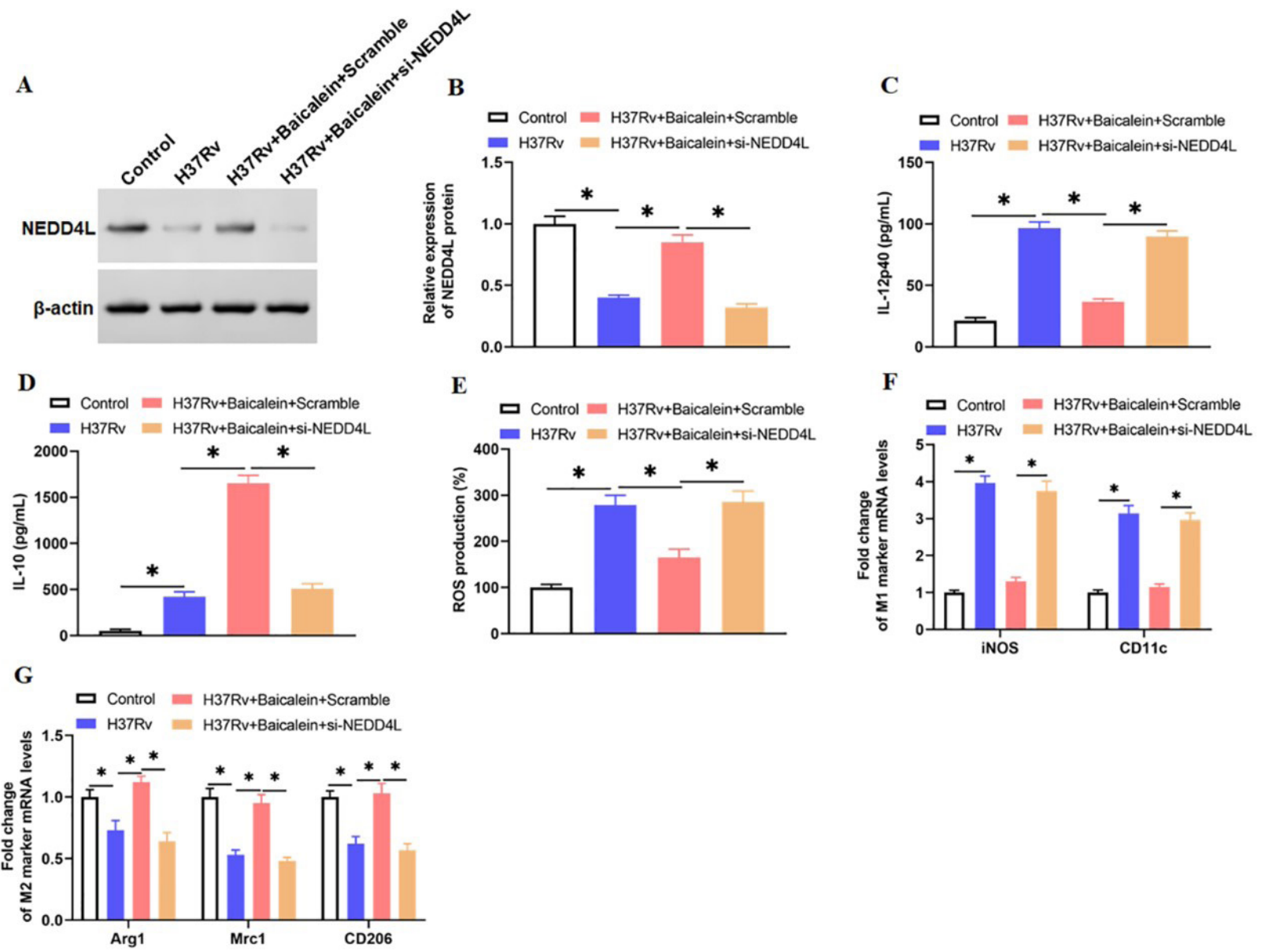

Figure 4. Baicalein regulates the expression of NEDD4L to promote M2 type polarization of macrophages after Mycobacterium tuberculosis infection. RAW264.7 cells were infected with $M$. tb H37Rv (MOI 1:10) for 4-6 h after incubated with $50 \mu \mathrm{M}$ baicalein for 24 h subsequently transfected with the NEDD4L interference vector (si-NEDD4L) or the corresponding negative control vector (Scramble) for $24 \mathrm{~h}$ with starvation medium. (A), (B) Western blotting was used to detect the protein expression of NEDD4L; (C), (D) ELISA measurement of IL-12p40 and IL-10 in cells; (E) The ROS level was detected by flow cytometry (DCFH-DA); (F), (G) RT-qPCR analyzed the mRNA levels of M1 markers (iNOS and CD11c) and M2 markers (Arg1, Mrc1 and CD206). Statistical significance was analyzed utilizing the Student's t-test. Values are exhibited as mean $\pm \mathrm{SEM}$. N $=6$, ${ }^{\star} P<0.05$.

\subsection{Baicalein regulates NEDD4L-mediated degradation of TLR2 ubiquitination}

We used the online database UbiBrowser to predict the proteins interacting with TLR2, and E3 ubiquitin ligase NEDD4L was one of them (Figure 5A), and the Co-IP experiment verified the binding of TLR2 and NEDD4L (Figure 5B). Proteasome inhibitors have always been the main means to determine the role of ubiquitin in different biological processes of the proteasome system, and MG132 is a potent and reversible synthetic peptide aldehyde proteasome inhibitor (Karpuz \& Çakir, 2021). In this study, we found that baicalein reduced the level of TLR2 protein in H37Rv-infected RAW264.7 cells in a dose-dependent manner, and MG132 reversed this effect (Figure 5C-E). Ubiquitination analysis experiments showed that the addition of baicalein increased the ubiquitination level of TLR2 protein, while MG132 significantly reduced the effect of baicalein (Figure 5F). These results suggested that baicalein may increase the ubiquitination of TLR2 in H37Rv-infected RAW264.7 cells by increasing the expression level of E3 ubiquitin ligase NEDD4L.

\subsection{NEDD4L overexpression increases the ubiquitination degradation of TLR2, inhibits the JNK pathway, promotes M2 type polarization of macrophages after M. tb H37Rv infection}

We found that compared with the control group, the expression level of NEDD4L protein was significantly reduced, and the expression levels of TLR2 and phosphorylated JNK proteins were significantly increased of RAW264.7 cells in H37Rv infection group (Figure 6A, B). Compared with the $\mathrm{H} 37 \mathrm{Rv}$ group, the upregulation of NEDD4L significantly increased the expression level of NEDD4L protein, and reduced the expression levels of TLR2 and phosphorylated JNK proteins in RAW264.7 cells, while proteasome inhibitor MG-132 reversed this effect (Figure 6A, B). Moreover, the addition of MG-132 increased IL-12p40 and ROS levels (Figure 6C, E), reduced the level of IL-10 (Figure 6D), and enhanced the polarization of macrophage M1 (Figure 6F), weakened the M2 polarization (Figure 6G). 

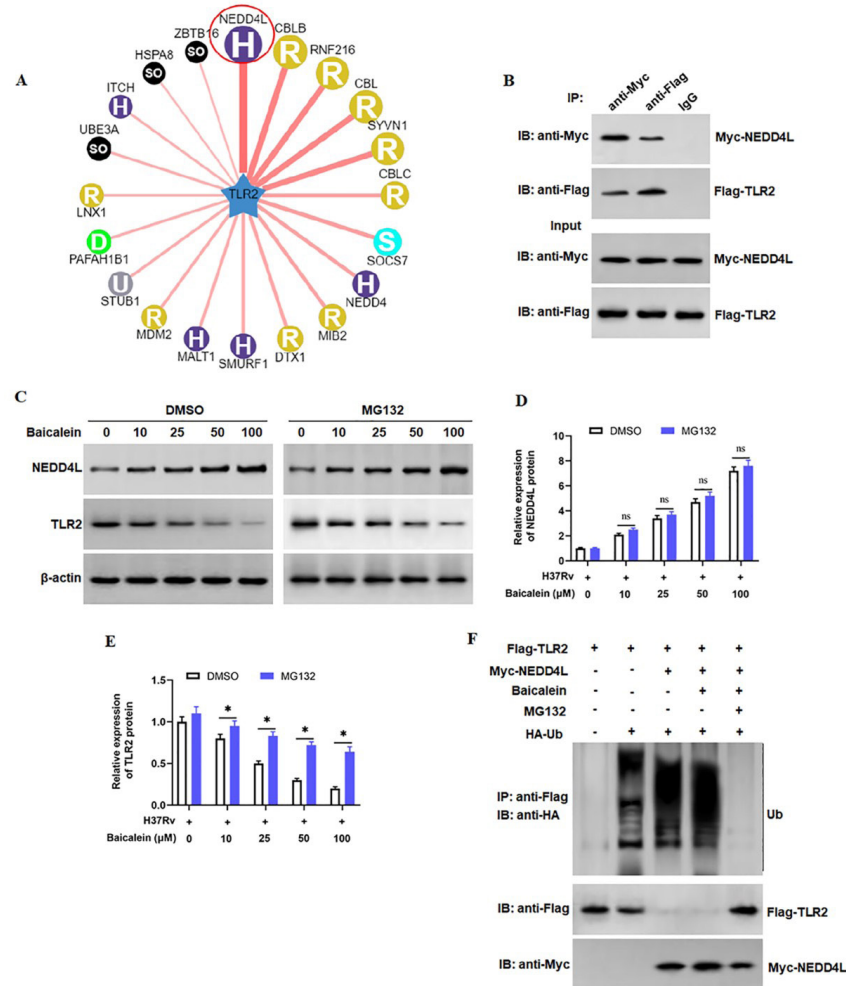

Figure 5. Baicalein regulates NEDD4L-mediated degradation of TLR2 ubiquitination. (A) The online database UbiBrowser predicted the binding of TLR2 and E3 ubiquitin ligase NEDD4L; (B) Co-IP experiment was used to verify the binding of TLR2 and E3 ubiquitin ligase NEDD4L. RAW264.7 cells were stimulated with $20 \mu \mathrm{M}$ proteasome inhibitor MG-132 for $4 \mathrm{~h}$ with starvation medium subsequently infected with M. $t b \mathrm{H} 37 \mathrm{Rv}$ (MOI 1:10) for 4-6 hours after incubated with $50 \mu \mathrm{M}$ baicalein for $24 \mathrm{~h}$; (C) Electropherogram of the protein expression levels of NEDD4L and TLR2 detected by Western blotting; (D), (E) Histogram of the protein expression levels of NEDD4L and TLR2 detected by Western blotting; (F) Ubiquitination analysis was used to detect the effect of baicalein on NEDD4L-mediated TLR2 ubiquitination. Statistical significance was analyzed utilizing the Student's t-test. Values are exhibited as mean \pm SEM. $\mathrm{N}=6$, ns $P>0.05,{ }^{\star} P<0.05$.

\subsection{Baicalein regulates NEDD4L-mediated TLR2 ubiquitination and inhibits JNK pathway}

To further investigate the regulation of baicalein on the JNK pathway on H37Rv-injected RAW264.7 cells, we injected H37Rv to RAW264.7 cells after treatment with baicalein, and we found that compared with the cells infected H37Rv only, the expression level of NEDD4L protein was significantly reduced (Figure 7A, B), and the expression levels of TLR 2 and phosphorylated JNK protein were significantly increased after baicalein treatment. Compared with the H37Rv combined with baicalein treatment group, the addition of JNK pathway inhibitor IQ 3 reduced the expression level of phosphorylated JNK protein (Figure 7A, B), but had no effect on the expression levels of NEDD4L and TLR2 proteins, reduced the levels of IL-12p40 and ROS (Figure 7C, E), which indicated that baicalein inhibited the JNK pathway by regulating NEDD4L-mediated ubiquitination of TLR2, thereby improving the inflammatory environment of RAW264.7 cells after H37Rv infection.

\subsection{Baicalein promotes $M 2$ polarization of macrophages to relieve pneumonia in mice caused by Mycobacterium tuberculosis infection}

We injected $1000 \pm 150 \mathrm{CFU}$ units of $\mathrm{H} 37 \mathrm{Rv}$ into the mouse nasal cavity on the 0 th and 7 th days to establish a mouse pneumonia model. After the virus infection, the mice were injected intraperitoneally with baicalein. On the 28th day, the lungs of the infected mice were taken out for pathological examination. The results showed that compared with the control mice, the lungs of the $\mathrm{H} 37 \mathrm{Rv}$ infected mice appeared obvious edema and pulmonary parenchymal hemorrhage (Figure 8A), the expression level of NEDD4L protein in lung tissue was significantly reduced, and the expression levels of TLR2 and phosphorylated JNK proteins in lung tissue was significantly increased (Figure 8C, D). Compared with the H37Rv infection group, pulmonary edema and pulmonary hemorrhage were significantly improved (Figure 8A), the expression level of NEDD4L protein in lung tissue was significantly reduced, and the expression levels of TLR2 and phosphorylated JNK proteins in lung tissue was significantly increased of mice in the baicalein treatment group (Figure 8C, D). In addition, baicalein treatment significantly increased the survival rate of mice (Figure $8 \mathrm{~B}$ ), reduced the relative weight of lung tissues (Figure $8 \mathrm{E}$ ), the bacterial burden of lung tissues (Figure 8F), the secretion of IL-12p40 (Figure $8 \mathrm{G}$ ) and ROS release (Figure 8I) of lung tissues of mice, inhibited the polarization of lung macrophages M1 (Figure 8J), and promoted M2 polarization (Figure $8 \mathrm{~K}$ ).

\section{Discussion}

M. $t b$ is a successful pathogen that causes about 1.5 million deaths in the world every year (Houben \& Dodd, 2016). Patients are transmitted by inhalation of atomized droplets and effective pulmonary infection is established. After the patient inhaled infectious $M$. $t b$, the bacteria were swallowed by alveolar macrophages patrolling the airway surface (Cohen et al., 2018), subsequently, the glycolipid on the surface of the $M$. $t b$ cell wall binding with TLR2 in macrophages to promote the secretion of Th-1 type cytokines (Harding \& Boom, 2010). A study reported that after the infection of macrophages with the Rv2770c protein of $M$. $t b$, it was found that the secretion of the pro-inflammatory cytokine interferon 12p40 (IL-12p40) was significantly increased, and it played a key regulatory role in Th- 1 cell immunity (Yu et al., 2017). Reactive oxygen species (ROS) are released under different physiological and pathological conditions(Vasconcelos et al., 2019), the secretion of pro-inflammatory inflammatory factors in macrophages increases the production of mitochondrial ROS and generates strong oxidative stress, thereby promoting the continuous opening of mitochondrial permeability transition pore complex (mPTP), leading to the destruction of membrane potential and ATP depletion, eventually leading to the necrosis of macrophages, and the bacteria in the cells are released to infect new macrophages, causing the infection to aggravate (Roca et al., 2019). In addition, a study detected granulomas in bovine lung lymph nodes infected with Mycobacterium bovis (M. bovis) and found that the level of macrophage M1 polarization marker molecule iNOS mRNA was significantly increased, while the level of macrophage M2 polarization marker 


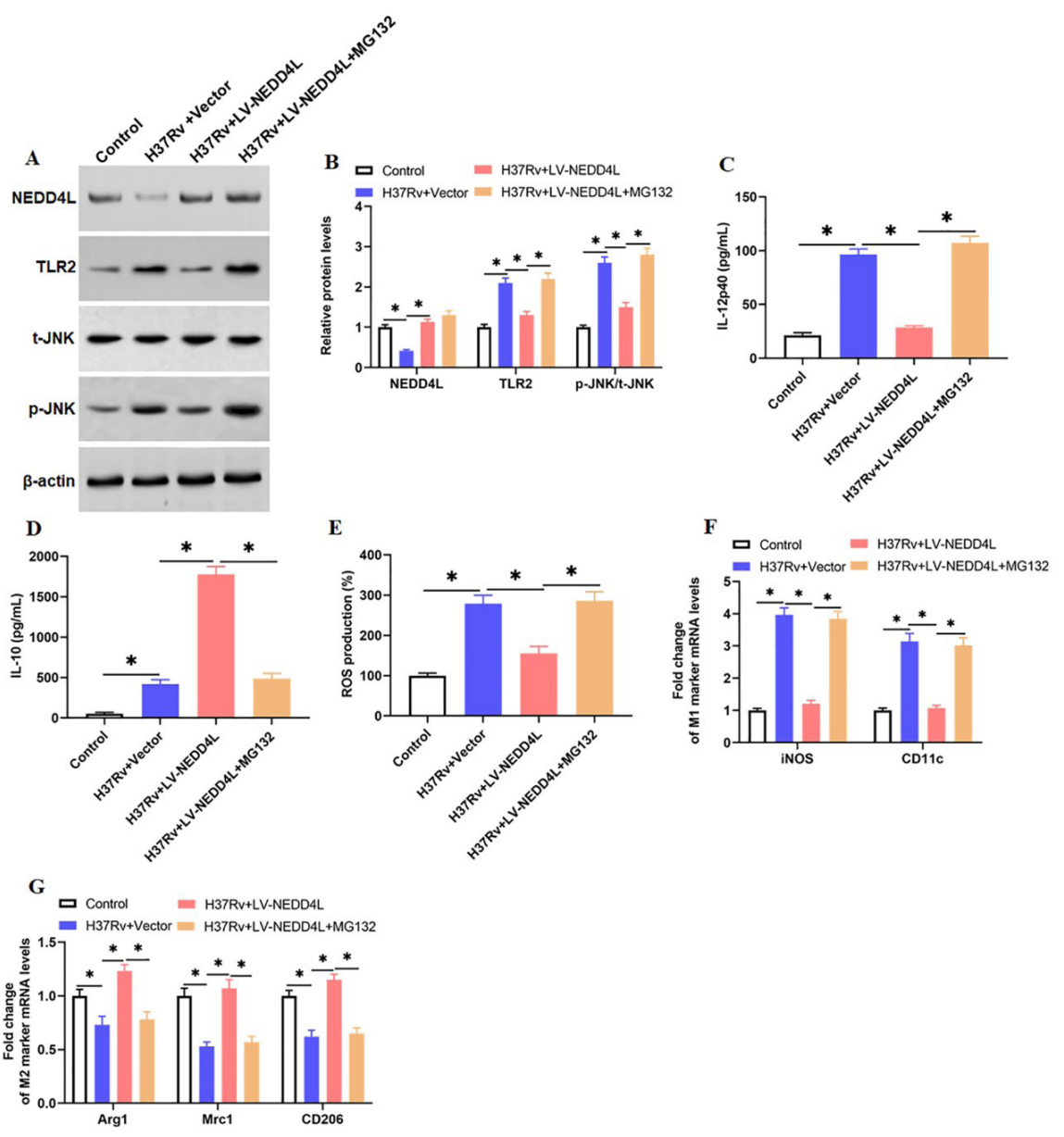

Figure 6. Upregulation of NEDD4L inhibits the JNK pathway by increasing the ubiquitination level of TLR2 and promotes M2 type polarization of macrophages after Mycobacterium tuberculosis infection. RAW264.7 cells were infected with M. tb H37Rv (MOI 1:10) for 4-6 hours after stimulated with $20 \mu \mathrm{M}$ proteasome inhibitor MG-132 for $4 \mathrm{~h}$ subsequently transfected with the NEDD4L lentiviral overexpression vector (LVNEDD4L) or the corresponding negative control vector (Vector) for $24 \mathrm{~h}$ with starvation medium. (A), (B) Western blotting was used to detect the proteins expression of NEDD4L, TLR2, total JNK and phosphorylated JNK; (C), (D) ELISA measurement of IL-12p40 and IL-10 levels in cells; (E) The ROS level was detected by flow cytometry (DCFH-DA); (F), (G) RT-qPCR analyzed the mRNA levels of M1 markers (iNOS and CD11c) and M2 markers (Arg1, Mrc1 and CD206). Statistical significance was analyzed utilizing the Student's t-test. Values are exhibited as mean \pm SEM. $\mathrm{N}=6,{ }^{\star} P<0.05$.

molecule Arg1 mRNA was significantly reduced, it is speculated that Arg1 expression can promote infection by competing with iNOS, thereby preventing the clearance of $M$. bovis by nitric oxide (Shu et al., 2014). Our research was consistent with it. We infected RAW264.7 cells and C57BL/6 mice with $M$. tb H37Rv, and found that the secretion levels of IL-12p40 and IL-10, ROS level and macrophage M1 polarization marker molecules (iNOS and $\mathrm{CD} 11 \mathrm{c}$ ) mRNA levels were significantly increased, while macrophages M2 polarization marker molecules (Arg1, Mrc1 and CD206) mRNA levels were significantly decreased both in RAW264.7 cells and mouse lung tissues.

Studies have been carried out in $293 \mathrm{~T}$ cells to be expressed together with cDNA encoding TLR4 or TLR9 and E3 ubiquitin ligase Triad3A, and it was found that Triad3A can completely degrade TLR4 and TLR9 proteins through ubiquitin protein (Chuang \& Ulevitch, 2004). A study found that the level of TLR2 in acute lung injury caused by Staphylococcus aureus infection may be reduced by the overexpression of RING E3 ubiquitin ligase PPP1R11. TLR2 immunoprecipitation and ubiquitin immunoblot analysis showed that PPP1R11 can specifically promote the polyubiquitination degradation of TLR2, and this process can be inhibited by the ubiquitination inhibitor MG132, moreover, in the mouse lung epithelial cell line, PPP1R11 can degrade TLR2 through ubiquitination to inhibit the secretion of TLR2-mediated pro-inflammatory inflammatory factors (Mckelvey et al., 2016). A genomic study found that the E3 ubiquitin ligase NEDD4L and TLR2 are associated with the ischemic heart disease phenotype (Makeeva et al., 2015). In addition, a study found that NEDD4L expression level was significantly down-regulated in mice with pneumonia caused by silver nanoparticles (Scoville et al., 2017). Our study found that in H37Rv-infected RAW264.7 cells and C57BL/6 mouse lung tissues, the expression level of NEDD4L protein was significantly downregulated. Meanwhile, we found that the binding of NEDD4L and TLR2 promoted the ubiquitination and degradation of TLR2. 


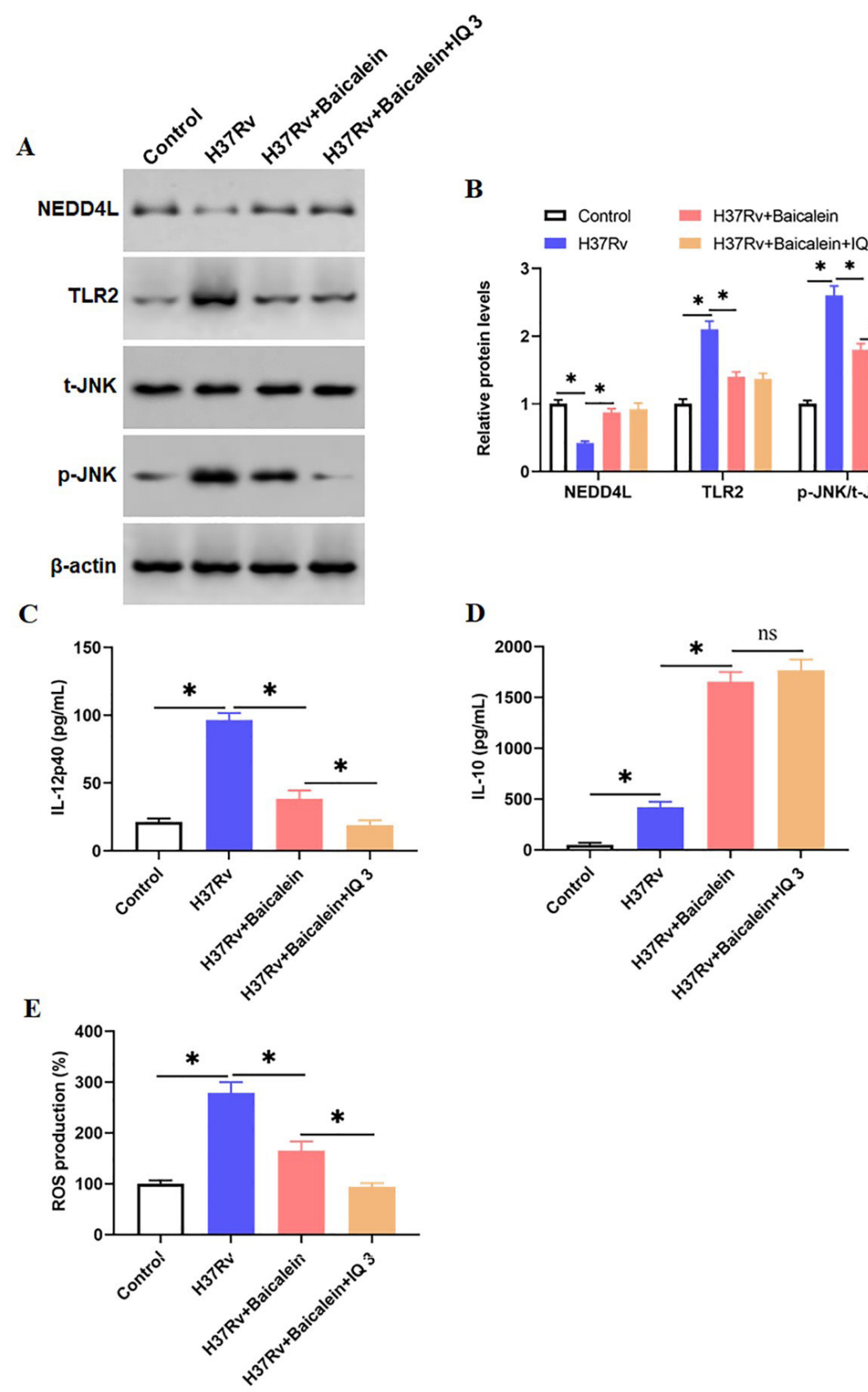

Figure 7. Baicalein regulates NEDD4L-mediated TLR2 ubiquitination and inhibits JNK pathway. RAW264.7 cells were infected with $M$. $t b$ H37Rv (MOI 1:10) for 4-6 h after incubated with $50 \mu \mathrm{M}$ baicalein for $24 \mathrm{~h}$ subsequently stimulated with $1.4 \mu \mathrm{M}$ JNK pathway inhibitor IQ 3 for $6 \mathrm{~h}$. (A), (B) Western blotting was used to detect the proteins expression of NEDD4L, TLR2, total JNK and phosphorylated JNK; (C), (D) ELISA measurement of IL-12p40 and IL-10 levels in cells; (E) The ROS level was detected by flow cytometry (DCFH-DA). Statistical significance was analyzed utilizing the Student's t-test. Values are exhibited as mean \pm SEM. $N=6,{ }^{\star} P<0.05$.

A study showed that $M . t b$ can promote the expression of fibrin connective tissue growth factor in human lung fibroblasts by increasing the phosphorylation of the c-Jun N-terminal kinase (JNK) pathway mediated by TLR2 to promote lung fibrosis (Lee et al., 2019). According to a study, baicalein can reduce the level of phosphorylated JNK protein, reduce the secretion of pro-inflammatory factors in macrophages, promote the M2 polarization of macrophages, and has a protective effect on cerebral ischemia caused by middle cerebral artery occlusion in rats (Yang et al., 2019). Baicalein is an effective component of the traditional Chinese medicine Scutellaria baicalensis Georgi. It has strong anti-inflammatory activity and can protect mice from death induced by Streptococcus pneumoniae by reducing the lung infiltration of inflammatory cells and inflammatory cytokines (Guo et al., 2019). Baicalein can also reduce the increase in liver TLR2, TLR3, TLR6 and TLR9 $\mathrm{mRNA}$ and protein expression levels induced by monocrotaline, and relieve liver inflammation (Zhang et al., 2016). Moreover, baicalein can also alleviate the neuronal damage induced by oxygen glucose ablation by reducing 
A

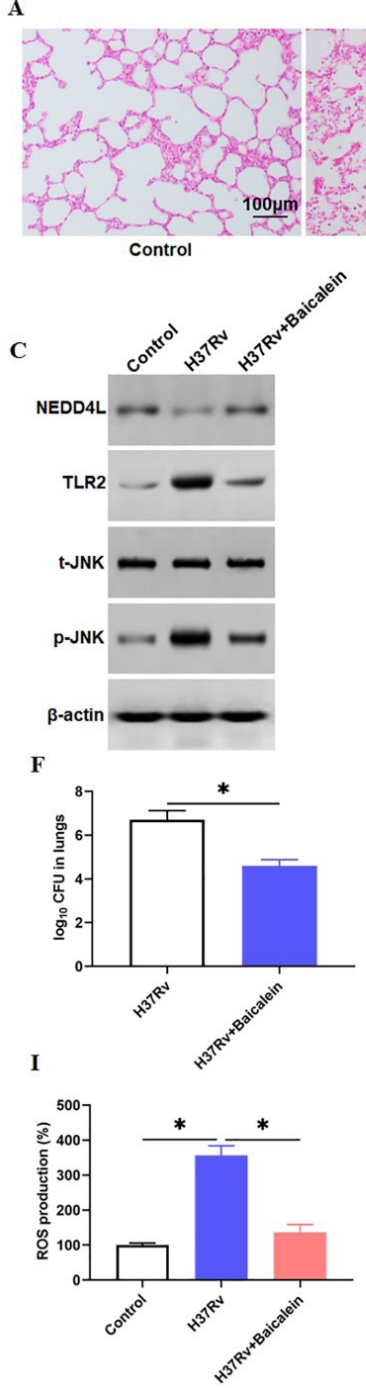

B
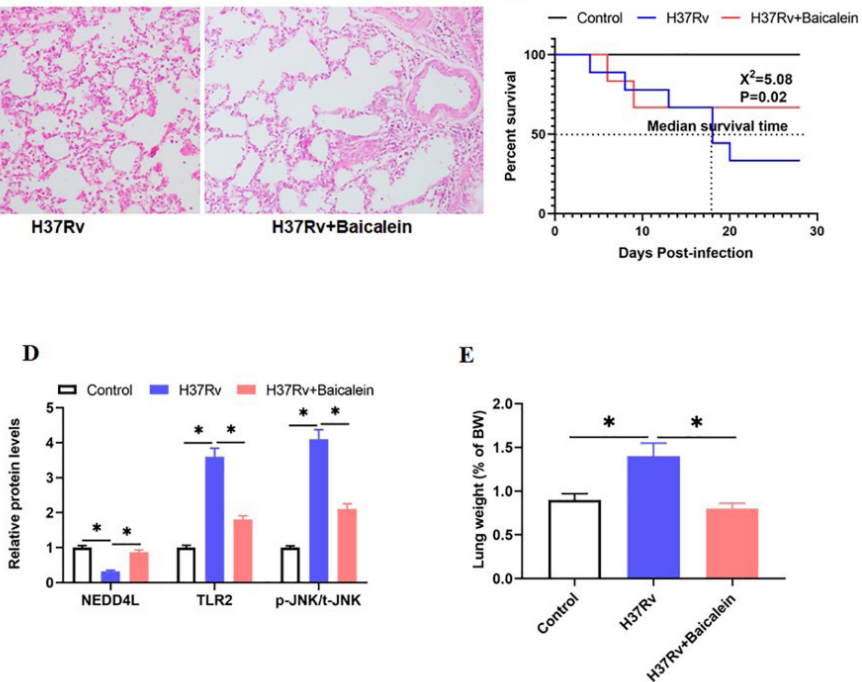

G
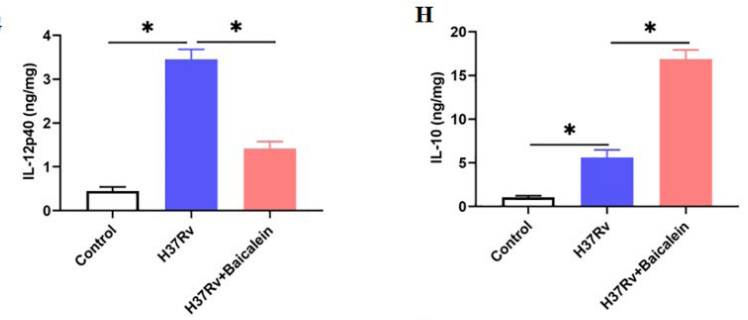

$\mathbf{K}$

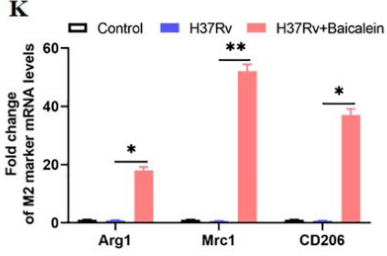

Figure 8. Baicalein promotes M2 polarization of macrophages to relieve pneumonia in mice caused by Mycobacterium tuberculosis infection. Mice in the M. tuberculosis infection group were intranasal infected with $M$. $t b \mathrm{H} 37 \mathrm{Rv}$ in 0 th and 7 th days with $1000 \pm 150 \mathrm{CFU} / \mathrm{mouse}$ after intraperitoneal infected light ketamine $(100 \mathrm{mg} / \mathrm{kg})$-xylasine $(10 \mathrm{mg} / \mathrm{kg})$ anesthesia. Mice in control group was injected with equal volume of saline. Mice in baicalein treatment group were injected intraperitoneally with $25 \mathrm{mg} / \mathrm{kg}$ baicalein after i'njected with bacterial every other day till $28 \mathrm{~d}$ after infected. (A) HE staining experiment was used to identify the histopathology of lungs in mice, images are magnified 200x normal from left to right; (B) Survival curve of mice; (C), (D) Western blotting was used to detect the proteins expression of NEDD4L, TLR2, total JNK and phosphorylated JNK in lung tissue homogenate; (E) Relative weight of mouse lungs; (F) Lung bacterial burden of mice; (G), (H) ELISA measurement of IL-12p40 and IL-10 levels in lung tissue homogenate; (I) The ROS level was detected by flow cytometry (DCFH-DA) in lung tissue homogenate; (J), (K) RT-qPCR analyzed the mRNA levels of M1 markers (iNOS and CD11c) and M2 markers (Arg1, Mrc1 and CD206) in lung tissue homogenate. Statistical significance was analyzed utilizing the Student's t-test. Values are exhibited as mean \pm SEM. $N=10,{ }^{\star} P<0.05,{ }^{\star *} P<0.01$.

the expression level of TLR2 protein (Li et al., 2012). A study through immunoprecipitation (IP) and Duolink test found that baicalein increased the binding of HDAC-1 and ubiquitin in leukemia cell lines, and the degradation of HDAC-1 induced by baicalein was blocked by the proteasome inhibitor MG-132, indicating that the degradation of HDAC-1 by baicalein may depend on the ubiquitin proteasome pathway (Yu et al., 2020). In this study, the results of ubiquitination analysis showed that baicalein can significantly promote NEDD4L-mediated ubiquitination of TLR2, and the addition of ubiquitination inhibitor MG132 can reverse the effect of baicalein. We also found that baicalein could significantly reduce the expression levels of TLR2 and phosphorylated JNK protein in
H37Rv-infected RAW264.7 cells and mouse lung tissue, and reduce the levels of pro-inflammatory cytokines IL-12p 40 and ROS, increase the level of anti-inflammatory cytokines IL-10, reduce the expression levels of iNOS and CD11c mRNA, increase the expression levels of Arg1, Mrc1 and CD206 mRNA, and reduce bacterial burden and inflammatory cell infiltration in mouse lung tissue.

In summary, our study found that baicalein has the protective effect of mouse pneumonia caused by $M$. $t b$, and we found that baicalein can promote the ubiquitination of TLR2 mediated by the E3 ubiquitin ligase NEDD4L, and promote $\mathrm{M} 2$ polarization of macrophages after $M$. $t b$ infection (Figure 9). 


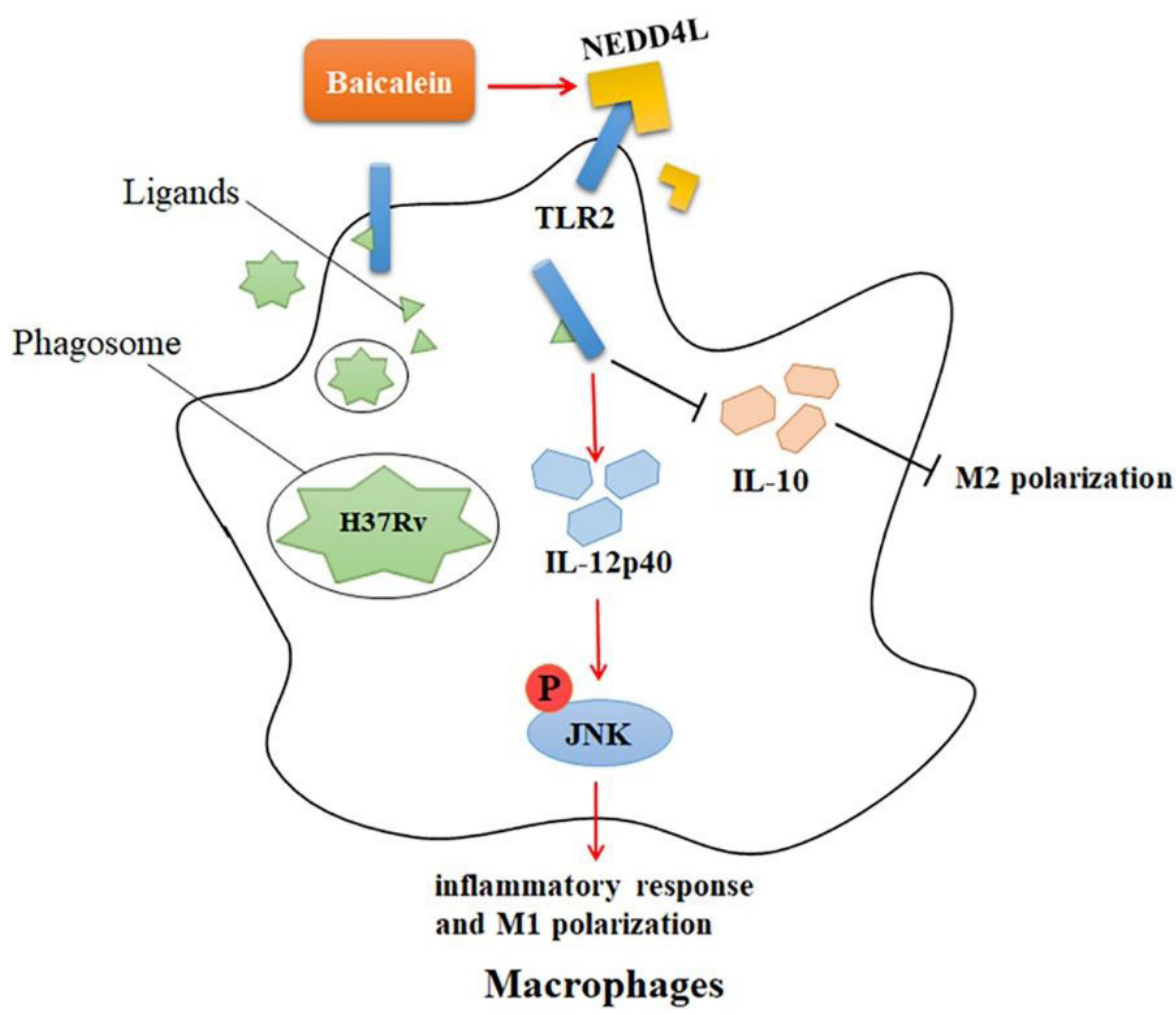

Figure 9. Baicalein promotes M2 polarization of macrophages by enhancing NEDD4L-mediated TLR2 ubiquitination, thereby resisting $M$. $t b$ infection. $M$. $t b$ is phagocytosed by macrophages and survives in phagosomes. $M$. $t b$ ligands, including lipoproteins and glycolipids, are recognized by TLR2 in macrophages, leading to increased JNK phosphorylation levels, promoting macrophage M1 polarization and increasing secretion levels of inflammatory factors, causing more intense inflammation reaction. The binding of $M$. $t b$ ligand to TLR2 inhibits the secretion of the anti-inflammatory factor IL-10 and inhibits macrophages M2 polarization. Baicalein promotes NEDD4L-mediated TLR2 ubiquitination by increasing the expression level of E3 ubiquitin ligase NEDD4L and promotes M2 polarization of macrophages, thereby enhancing the ability of macrophages to resist $M$. $t b$ infection.

\section{Abbreviations}

M. tb: Mycobacterium tuberculosis. DMEM: Dulbecco's Modified Eagle Medium. PBS: phosphate buffered saline. FBS: Fetal Bovine Serum. ROS: reactive oxygen species. TB: tuberculosis. TLR2: toll-like receptor 2. NEDD4L: Neural precursor cell expressed developmentally downregulated gene 4-like.

\section{Ethical approval}

All patients gave informed consent and signed an informed consent form. All samples obtained in this study were approved by the ethics committee of the Xi'an Peihua University Medical School and abided by the ethical guidelines of the Declaration of Helsinki. All animal experiments comply with the guidelines for the care and use of laboratory animals established by the National Institutes of Health (Bethesda, Maryland, USA).

\section{Conflict of interest}

The authors declare that there is no conflict of interest regarding the publication of this paper.

\section{Author contributions}

Min Shi designed the study. Min Shi wrote the manuscript. Pengyi Yin and Xiaobo Guo performed the experiments. Qian Li and Lin Sun collected and analyzed data. Xiaohua Cao revised and proofread the manuscript.

\section{References}

Bao, H., Zhao, D., \& Du, X. (2021). Extraction of baicalin from Scutellaria baicalensis Georgi and its alleviative effect on acute respiratory distress syndrome in rats. Food Science and Technology, [Ahead of print].

Chuang, T. H., \& Ulevitch, R. J. (2004). Triad3A, an E3 ubiquitinprotein ligase regulating Toll-like receptors. Nature Immunology, 5(5), 495-502. http://dx.doi.org/10.1038/ni1066. PMid:15107846.

Cohen, S. B., Gern, B. H., Delahaye, J. L., Adams, K. N., Plumlee, C. R., Winkler, J. K., Sherman, D. R., Gerner, M. Y., \& Urdahl, K. B. (2018). Alveolar macrophages provide an early Mycobacterium tuberculosis niche and initiate dissemination. Cell Host \& Microbe, 24(3), 439446. http://dx.doi.org/10.1016/j.chom.2018.08.001. PMid:30146391.

Collins, G. A., \& Goldberg, A. L. (2017). The Logic of the 26S Proteasome. Cell, 169(5), 792-806. http://dx.doi.org/10.1016/j.cell.2017.04.023. PMid:28525752.

Dinda, B., Dinda, S., Dassharma, S., Banik, R., Chakraborty, A., \& Dinda, M. (2017). Therapeutic potentials of baicalin and its aglycone, baicalein against inflammatory disorders. European Journal of Medicinal Chemistry, 131, 68-80.

Eyking, A., Ferber, F., Köhler, S., Reis, H., \& Cario, E. (2019). TRIM58 restrains intestinal mucosal inflammation by negatively regulating TLR2 in myeloid cells. Journal of Immunology, 203(6), 1636-1649. http://dx.doi.org/10.4049/jimmunol.1900413. PMid:31383741.

Gilleron, M., Nigou, J., Nicolle, D., Quesniaux, V., \& Puzo, G. (2006). The acylation state of mycobacterial lipomannans modulates innate 
immunity response through toll-like receptor 2. Chemistry \& Biology, 13(1), 39-47. http://dx.doi.org/10.1016/j.chembiol.2005.10.013. PMid:16426970.

Guo, Q., Liu, G., Xiaobin, L., \& Qingsong, H. (2019). Baicalein exerts a protective role in pneumonia caused by Streptococcus pneumoniae. Frontiers in bioscience, 24, 849-858.

Harding, C. V., \& Boom, W. H. (2010). Regulation of antigen presentation by Mycobacterium tuberculosis: a role for Toll-like receptors. Nature Reviews. Microbiology, 8(4), 296-307. http://dx.doi.org/10.1038/ nrmicro2321. PMid:20234378.

Houben, R., \& Dodd, P. (2016). The global burden of latent tuberculosis infection: a re-estimation using mathematical modelling. PLoS Medicine, 13(10), e1002152. http://dx.doi.org/10.1371/journal. pmed.1002152. PMid:27780211.

Huang, Y., Wan, B., Huang, Z., \& Lin, M. (2021). Effect of Jianpibufei plaster on chronic obstructive pulmonary disease in mice. Food Science and Technology. [Ahead of print]. http://dx.doi.org/10.1590/ fst. 39320 .

Hunter R.L. (2018). The pathogenesis of tuberculosis: the early infiltrate of post-primary (adult pulmonary) tuberculosis: a distinct disease entity. Frontiers in Immunology, 9, 2108.

Karpuz, B., \& Çakir, Ö. (2021). Effect of proteasome inhibitor MG132 on the expression of oxidative metabolism related genes in tomato. Food Science and Technology. [Ahead of print]. http://dx.doi. org/10.1590/fst.52420.

Lee, H. S., Hua, H. S., Wang, C. H., Yu, M. C., Chen, B. C., \& Lin, C. H. (2019). Mycobacterium tuberculosis induces connective tissue growth factor expression through the TLR2-JNK-AP-1 pathway in human lung fibroblasts. The FASEB Journal, 33(11), 12554-12564. http://dx.doi.org/10.1096/fj.201900487R. PMid:31451010.

Li H., Hu J., Zhao S., Yuan Z., Wan H., Lei F., Ding Y., Xing D., \& Du, L. (2012). Comparative study of the effect of baicalin and its natural analogs on neurons with oxygen and glucose deprivation involving innate immune reaction of TLR2/TNF. BioMed Research International, 2012, 267890.

Makeeva, O. A., Sleptsov, A. A., Kulish, E. V., Barbarash, O. L., Mazur, A. M., Prokhorchuk, E. B., Chekanov, N. N., Stepanov, V. A., \& Puzyrev, V. P. (2015). Genomic study of cardiovascular continuum comorbidity. Acta Naturae, 7(3), 89-99. http://dx.doi.org/10.32607/207582512015-7-3-89-99. PMid:26483964.

Mckelvey A.C., Lear T.B., Dunn S.R., John E., Londino J.D., Bednash J.S., Yingze Z., Mcverry B.J., Yuan L., \& Chen B.B. (2016). RING finger E3 ligase PPP1R11 regulates TLR2 signaling and innate immunity. Elife, 5, e18496.

Meyer-Schwesinger, C. (2019). The ubiquitin-proteasome system in kidney physiology and disease. Nature Reviews. Nephrology, 15(7), 393-411. http://dx.doi.org/10.1038/s41581-019-0148-1. PMid:31036905.
Repique, C. J., Li, A., Brickey, W. J., Ting, J. P., Collins, F. M., \& Morris, S. L. (2003). Susceptibility of mice deficient in the MHC class II transactivator to infection with Mycobacterium tuberculosis. Scandinavian Journal of Immunology, 58(1), 15-22. http://dx.doi. org/10.1046/j.1365-3083.2003.01266.x. PMid:12828554.

Roca, F. J., Whitworth, L. J., Redmond, S., Jones, A. A., \& Ramakrishnan, L. (2019). TNF Induces pathogenic programmed macrophage necrosis in tuberculosis through a mitochondrial-lysosomal-endoplasmic reticulum circuit. Elsevier Sponsored Documents, 178(6), 13441361. http://dx.doi.org/10.1016/j.cell.2019.08.004. PMid:31474371.

Scoville, D. K., Botta, D., Galdanes, K., Schmuck, S. C., White, C. C., Stapleton, P. L., Bammler, T. K., MacDonald, J. W., Altemeier, W. A., Hernandez, M., Kleeberger, S. R., Chen, L., Gordon, T., \& Kavanagh, T. J. (2017). Genetic determinants of susceptibility to silver nanoparticle-induced acute lung inflammation in mice. The FASEB Journal, 31(10), 4600-4611. http://dx.doi.org/10.1096/ fj.201700187R. PMid:28716969.

Shi, L., Jiang, Q., Bushkin, Y., Subbian, S., \& Tyagi, S. (2019). Biphasic dynamics of macrophage immunometabolism during mycobacterium tuberculosis infection. mBio, 10(2), e02550-18. PMid:30914513.

Shu, D., Heiser, A., Wedlock, D. N., Luo, D., Lisle, G. W., \& Buddle, B. M. (2014). Comparison of gene expression of immune mediators in lung and pulmonary lymph node granulomas from cattle experimentally infected with Mycobacterium bovis. Veterinary Immunology and Immunopathology, 160(1-2), 81-89. http://dx.doi.org/10.1016/j. vetimm.2014.03.017. PMid:24852075.

Vasconcelos, F.M., Silva, H., Poso, S., Barroso, M.V., \& Valena, S.S. (2019). Probiotic Prato cheese attenuates cigarette smoke-induced injuries in mice. Food Research International, 123, 697-703.

Yang, S., Wang, H., Yang, Y., Wang, R., \& Du, G. (2019). Baicalein administered in the subacute phase ameliorates ischemia-reperfusioninduced brain injury by reducing neuroinflammation and neuronal damage. Biomedicine \& Pharmacotherapy, 117, 109102.

Yu, X., Li, H., Hu, P., Qing, Y., Wang, X., Zhu, M., Wang, H., Wang, Z., Xu, J., Guo, Q., \& Hui, H. (2020). Natural HDAC-1/8 inhibitor baicalein exerts therapeutic effect in CBF-AML. Clinical and Translational Medicine, 10(4), e154. http://dx.doi.org/10.1002/ ctm2.154. PMid:32898337.

Yu, Z., Zhang, C., Zhou, M., Li, Q., Li, H., Duan, W., Li, X., Feng, Y., \& Xie, J. (2017). Mycobacterium tuberculosis PPE44 (Rv2770c) is involved in response to multiple stresses and promotes the macrophage expression of IL-12 p40 and IL-6 via the p38, ERK, and NF-kappa B signaling axis. International Immunopharmacology, 50, 319-329. http://dx.doi.org/10.1016/j.intimp.2017.06.028. PMid:28743081.

Zhang, J., Sheng, Y., Shi, L., Zheng, Z., Chen, M., Lu, B., \& Ji, L. (2016). Quercetin and baicalein suppress monocrotaline-induced hepatic sinusoidal obstruction syndrome in rats. European Journal of Pharmacology, 795, 160-168. 